\title{
Generalized Bernoulli Numbers and $m$-Regular Primes
}

\author{
By Fred H. Hao and Charles J. Parry
}

\begin{abstract}
A prime $p$ is defined to be $m$-regular if $p$ does not divide the class number of a certain abelian number field. Several different characterizations are given for a prime to be $m$-regular, including a description in terms of the generalized Bernoulli numbers. A summary is given of two computations which determine the $m$-regularity or $m$-irregularity of primes $p$ for certain values of $m$ and $p$.
\end{abstract}

In an earlier article [3], we defined $m$-regular primes and showed under certain simple congruence conditions that the Fermat equation of exponent $p$ has no solutions in the field $Q(\sqrt{m})$ when $p$ is an $m$-regular prime. In this note we give several equivalent conditions for a prime to be $m$-regular. In particular, it is possible to describe $m$-regularity by means of the generalized Bernoulli numbers.

The following notation will be used throughout this article.

$Q:$ the field of rational numbers.

$Z$ : ring of rational integers.

$m:$ a square-free integer, $m \neq 1$.

$p$ : an odd prime.

$\zeta$ : a primitive $p$ th root of unity.

$k=Q(\sqrt{m})$ : quadratic number field.

$L=Q\left(\zeta+\zeta^{-1}\right)$.

$K=Q(\zeta, \sqrt{m})$.

$K_{1}$ : the maximal real subfield of $K$.

$K_{3}=Q(\zeta)$ : the $p$ th cyclotomic field.

$K_{2}$ : the totally imaginary quadratic extension of $L$ contained in $K$ with $K_{2} \neq K_{3}$.

$h()$ : the class number of the field ( ).

() : the character group of the field ( ).

$\chi$ : a character in $\hat{K}$.

$f(\chi)$ : the conductor of $\chi$.

$d=|m|$ or $4|m|:$ the conductor of $k$.

The prime $p$ is defined to be $m$-regular if $p$ does not divide $h(K)$. Since $m$ may be replaced with the square-free kernel of $(-1)^{(p-1) / 2} \cdot p m$, we may assume that $(m, p)=1$.

Received January 17, 1983; revised August 4, 1983 and October 11, 1983.

1980 Mathematics Subject Classification. Primary 12A50, 12A35, 12A15. 
Theorems 1 and 2 of Parry [6] show that a regular prime $p$ is $m$-irregular if and only if $p \mid h\left(K_{2}\right)$ and Hasse [4, p. 12] shows that $p \mid h\left(K_{2}\right)$ if and only if $p \mid h^{*}$ where

$$
h^{*}=Q^{*} \omega \prod_{\substack{\chi \in \hat{K}_{2} \\ \chi(-1)=-1}} \frac{1}{2 f(\chi)} \sum_{t=1}^{f(\chi)}(-\chi(t) t) .
$$

Here $Q^{*}$ is the index of the unit group of $L$ in the unit group of $K_{2}$ and $\omega$ is the number of roots of unity in $K_{2}$. Now $Q^{*}$ is easily seen to be a power of 2 and since $K_{3}$ is not a subfield of $K_{2}, p$ does not divide $\omega$. Thus $\left(p, Q^{*} \omega\right)=1$ and $p \mid h^{*}$ if and only if

$$
\prod_{\substack{x \in \hat{K}_{2} \\ x(-1)=-1}} G_{\chi} \equiv 0 \quad(\bmod p)
$$

as a $p$-adic integer where

$$
G_{\chi}=\frac{1}{f(\chi)} \sum_{t=1}^{f(\chi)} \chi(t) t=B_{1, \chi}
$$

by Iwasawa [5, p. 14] where $B_{1, \chi}$ is the first generalized Bernoulli number.

If $\chi_{1}$ is the generator of $\hat{K}_{3}$, then $\hat{L}=\left\langle\chi_{1}^{2}\right\rangle$. If $\chi_{2}$ is a generator of $\hat{k}$, we will describe $\hat{K}_{2}$ in terms of $\chi_{1}$ and $\chi_{2}$. When $m<0, K_{2}=L k$ with $L \cap k=Q$. Therefore, $\hat{K}_{2}=\hat{L} \times \hat{k}$, and so

$$
\hat{K}_{2}=\left\{\chi_{1}^{2 j} \chi_{2}^{l} \mid 0 \leqslant j \leqslant(p-3) / 2,0 \leqslant l \leqslant 1\right\} .
$$

If $m>0$, then $K=K_{3} k$ with $K_{3} \cap k=Q$, so $\hat{K} \simeq \hat{K}_{3} \times \hat{k}$. Because $\left[\hat{K}: \hat{K}_{2}\right]=2$ and $\hat{K}_{1}=\hat{L} \times \hat{k}, \hat{K}_{3}=\left\langle\chi_{1}\right\rangle$, it follows that

$$
\hat{K}_{2}=\left\{\chi_{1}^{2 j}, \chi_{1}^{2 j+1} \chi_{2} \mid 0 \leqslant j \leqslant(p-3) / 2\right\} .
$$

Since $\chi_{1}$ is odd and $\chi_{2}$ is odd exactly when $m<0$, the odd characters of $\hat{K}_{2}$ have the form $\chi_{1}^{n} \chi_{2}$, where $0 \leqslant n \leqslant p-2$ and $n$ is odd or even according as $m$ is positive or negative. Note that when $m<0$ and $n=0, f\left(\chi_{2}\right)=d$ and

$$
G_{\chi_{2}}=\frac{1}{d} \sum_{t=1}^{d} \chi_{2}(t) t=-h(k) .
$$

Moreover, for $n>0, f\left(\chi_{1}^{n} \chi_{2}\right)=p d$.

THEOREM 1. Let $p$ be a prime with $(p, m)=1$ and $\chi_{2}(a)=(D / a)$ be the Kronecker symbol where $D$ is the discriminant of $Q(\sqrt{m})$. Then $p$ is m-regular if and only if

(i) $p$ is regular and

(ii) none of the numerators $B_{n+1, \chi_{2}}$ is divisible by $p$ where $B_{n+1, \chi_{2}}$ is the $(n+1)$ st generalized Bernoulli number and $0 \leqslant n \leqslant p-2$ with $n$ odd or even according as $m$ is positive or negative.

Proof. See Section 2 of Iwasawa [5] or Washington [9, pp. 30-31] for definitions and basic properties of generalized Bernoulli numbers. If $p$ is irregular, then class field theory shows $p$ is $m$-irregular. If $p$ is regular, then the above remarks show $p$ is $m$-irregular if and only if $B_{1, \chi} \equiv 0(\bmod p)$ for some character $\chi=\chi_{1}^{n} \chi_{2}$ with $n$ as 
described in (ii). Exercise 7.5 of Washington [9, p. 141] shows that $B_{n+1, \chi_{2}} /(n+1)$ $\equiv B_{1, \chi}(\bmod p)$. Since $p+(n+1)$, the theorem follows immediately.

COROLlaRY. Let $p$ be an odd regular prime with $(p, m)=1$ and $\chi=\chi_{2}$ be the nontrivial character of the field $k$. Then $p$ is $m$-irregular if and only if there exists $a$ natural number $n$ with $n=1$ or $n \neq \equiv 1(\bmod (p-1))$ for $n>1$ and $n \equiv \delta_{\chi}(\bmod 2)$ such that $B_{n, \chi} / n \equiv 0(\bmod p)$, where $\delta_{\chi}=0$ or 1 according as $m$ is positive or negative.

Proof. If $p$ is $m$-irregular, the above theorem shows there exists $n$ with $1 \leqslant n \leqslant$ $p-1$ and $n \equiv \delta_{\chi}(\bmod 2)$ such that

$$
B_{n, \chi} \equiv 0(\bmod p), \quad \text { so } B_{n, \chi} / n \equiv 0(\bmod p) .
$$

Conversely, assume $B_{n, \chi} / n \equiv 0(\bmod p)$ for some natural number $n$ satisfying our hypothesis. If $n=1$, then as seen in the proof of the theorem,

$$
G_{\chi} \equiv B_{1, \chi} \equiv 0 \quad(\bmod p)
$$

and $p$ is $m$-irregular. Otherwise, $n \not \equiv 1(\bmod (p-1))$, and so there exists an integer $l$ with $2 \leqslant l \leqslant p-1$ such that $n \equiv l(\bmod (p-1))$. Theorem 5 of Carlitz [1] shows that

$$
B_{n, \chi} / n \equiv B_{l, \chi} / l \quad(\bmod p),
$$

and so $B_{l, \chi} \equiv 0(\bmod p)$. Since $p-1$ is even, $l \equiv n \equiv \delta_{\chi}(\bmod 2)$ and our theorem shows $p$ is $m$-irregular.

Remark. The hypothesis that $n \not \equiv 1(\bmod (p-1))$ for $n>1$ in the above corollary can be dropped when $m>0$ or when $m<0$ and $\chi(p)=(D / p)=-1$.

Proof. When $m$ is positive, $\delta_{\chi}$ is 0 and hence $n$ must be even. Hence $n \not \equiv 1$ $(\bmod (p-1))$ is automatically satisfied.

Suppose now $m$ is negative, Theorem 6 of Carlitz [1] shows for $n \equiv 1(\bmod (p-1))$ that

$$
B_{n, \chi} / n \equiv(1-\chi(p)) B_{1, \chi}(\bmod p)
$$

Thus, if $\chi(p)=-1$, then $B_{n, \chi} / n \equiv 0(\bmod p)$ if and only if $B_{1, \chi} \equiv 0(\bmod p)$, which implies that $p$ is $m$-irregular.

Let $g$ be a primitive root modulo $p$ chosen so that $g^{p-1} \equiv 1\left(\bmod p^{2}\right)$. Moreover, let $\eta$ be a primitive $(p-1)$ st root of unity such that $\chi_{1}(g)=\eta$. The following result is easily proved using standard techniques of algebraic number theory.

LEMMA 1. There exists a prime divisor $P$ of $p$ in $Q(\eta)$ such that $g \equiv \eta\left(\bmod P^{2}\right)$.

Assume now that $p+h(k)$. Then $p \mid \Pi_{\chi \in \hat{K}_{2}: \chi \text { odd }} G_{\chi}$ if and only if there exists $n$ with $0<n<p-1$ with $n$ odd or even according as $m$ is positive or negative and $\chi=\chi_{1}^{n} \chi_{2}$ such that $G_{\chi} \equiv 0(\bmod P)$ for the prime divisor $P$ of $p$ in $Q(\eta)$.

LEMMA 2. If $0<n<p-1, \chi=\chi_{1}^{n} \chi_{2}$ and $P$ is the prime ideal defined above, then

$$
G_{\chi} \equiv 0(\bmod P) \quad \text { iff } \quad \sum_{t=1}^{d-1} \chi_{2}(t) \sum_{u=0}^{c t-1} u^{n} \equiv 0(\bmod p),
$$

where $c d \equiv 1(\bmod p)$. 
Proof. Let $B_{i}(i \geqslant 0)$ denote the ordinary Bernoulli numbers and

$$
B_{n}(X)=\sum_{i=0}^{n}\left(\begin{array}{c}
n \\
i
\end{array}\right) B_{i} X^{n-i}
$$

denote the $n$th Bernoulli polynomial. Exercise 4.1 of Washington $[9$, p. 45] shows that

$$
\sum_{u=0}^{c t-1} u^{n}=\left(\frac{1}{n+1}\right)\left[B_{n+1}(c t)-B_{n+1}(0)\right] .
$$

Since $1<n+1<p$, the von Staudt-Clausen Theorem shows that none of $B_{0}, B_{1}, \ldots, B_{n}$ has denominators divisible by $p$. Thus

$$
B_{n+1}(c t)-B_{n+1}(0) \equiv B_{n+1}(t / d)-B_{n+1}(0) \quad(\bmod p) .
$$

Hence

$$
\begin{aligned}
\sum_{t=1}^{d-1} \chi_{2}(t) \sum_{u=0}^{c t-1} u^{n} & \equiv\left(\frac{1}{n+1}\right) \sum_{t=1}^{d-1} \chi_{2}(t)\left[B_{n+1}(t / d)-B_{n+1}(0)\right] \quad(\bmod p) \\
& \equiv\left(\frac{1}{n+1}\right) \sum_{t=1}^{d-1} \chi_{2}(t) B_{n+1}(t / d) \quad(\bmod p) \\
& \equiv \frac{d^{-n}}{n+1} B_{n+1, \chi_{2}} \equiv \frac{1}{d^{n}} B_{1, \chi} \equiv \frac{1}{d^{n}} G_{\chi}(\bmod p)
\end{aligned}
$$

The final three congruences follow from Proposition 4.1 of Washington [9, p. 31] and arguments used in the proof of Theorem 1. Since $p+d$, the lemma follows immediately.

THEOREM 2. Let $p$ be an odd regular prime and $\chi=\chi_{2}$ be the nontrivial character of the field $k=Q(\sqrt{m})$ with conductor $f=d$ where $(m, p)=1$. Then $p$ is $m$-regular if and only if

$$
\sum_{j=1}^{p} S_{n}(j) A_{j k} \not \equiv 0 \quad(\bmod p)
$$

for all $n$ with $1 \leqslant n \leqslant p-1$ and $n \equiv 1+\delta_{\chi}(\bmod 2)$. Here

$$
S_{n}(j)=\sum_{u=0}^{j-1} u^{n} \text { and } A_{n}=\sum_{\substack{t=1 \\ t=n(\bmod p)}}^{f} \chi(t) .
$$

Proof. The remarks preceding Theorem 1 combined with Lemma 2 show that $p$ is $m$-regular if and only if

$$
p+h(k) \text { for } m \text { negative }
$$

and

$$
\sum_{t=1}^{f} \chi(t) \sum_{u=0}^{c t-1} u^{n} \neq 0 \quad(\bmod p)
$$

for all $n$ with $1 \leqslant n \leqslant p-2$ and $n \equiv 1+\delta_{\chi}(\bmod 2)$. Here $c \equiv f^{-1}(\bmod p)$.

Recall $S_{n}(p)=\sum_{u=0}^{p-1} u^{n} \equiv 0(\bmod p)$ if $n$ is not divisible by $p-1$. Hence, for such $n, i \equiv j(\bmod p)$ implies

$$
S_{n}(i) \equiv S_{n}(j) \quad(\bmod p) .
$$


Moreover, $t \equiv j f(\bmod p)$ implies $c t-1 \equiv c j f-1 \equiv j-1(\bmod p)$. Thus

$$
\begin{aligned}
\sum_{t=1}^{f} \chi(t) \sum_{u=0}^{c t-1} u^{n} & =\sum_{j=1}^{p}\left(\sum_{\substack{t=1 \\
t \equiv j f(\bmod p)}}^{f} \chi(t) \sum_{u=0}^{c t-1} u^{n}\right) \\
& \equiv \sum_{j=1}^{p}\left(\sum_{\substack{t=1 \\
t \equiv j f(\bmod p)}}^{f} \chi(t) \sum_{u=0}^{j-1} u^{n}\right) \equiv \sum_{j=1}^{p} S_{n}(j) A_{j f}(\bmod p)
\end{aligned}
$$

When $m<0$, the additional condition $p+h(k)$ is equivalent to

$$
\frac{1}{f} \sum_{t=1}^{f} \chi(t) t \neq 0 \quad(\bmod p)
$$

Since

$$
S_{p-1}(j)=\sum_{u=0}^{j-1} u^{p-1} \equiv j-1 \quad(\bmod p) \quad \text { and } \quad \sum_{j=1}^{p} A_{j f}=\sum_{a=1}^{f} \chi(a)=0
$$

it follows that

$$
\begin{aligned}
\frac{1}{f} \sum_{t=1}^{f} \chi(t) t & \equiv \sum_{j=1}^{p}(j) A_{j f}(\bmod p) \\
& \equiv \sum_{j=1}^{p}(j-1) A_{j f} \equiv \sum_{j=1}^{p} S_{p-1}(j) A_{j f} \quad(\bmod p)
\end{aligned}
$$

The following result is easily verified.

LeMma 3. For any $n, A_{f-n}=\chi(-1) A_{n}$.

LEMMA 4. If $f=p g+i$ with $1 \leqslant i \leqslant p-1$, then

$$
A_{0}=\chi(p) \sum_{t=0}^{g} \chi(t)
$$

and

$$
A_{p-i}=\chi(p) \sum_{t=g+1}^{2 g+j} \chi(t) \quad \text { where } j= \begin{cases}0 & \text { if } 2 i<p \\ 1 & \text { if } 2 i>p\end{cases}
$$

When $2 i<p$,

$$
A_{p-2 i}=\chi(p) \sum_{t=2 g+1}^{3 g+l} \chi(t), \quad \text { where } l= \begin{cases}0 & \text { if } 3 i \leqslant p \\ 1 & \text { if } 3 i>p\end{cases}
$$

and when $2 i>p$,

$$
A_{2 p-2 i}=\chi(p) \sum_{t=2 g+2}^{3 g+s+1} \chi(t), \quad \text { where } s= \begin{cases}0 & \text { if } \frac{3}{2} i \leqslant p \\ 1 & \text { if } \frac{3}{2} i>p\end{cases}
$$


Proof. For any integer $n$ with $0 \leqslant n<p$, set

$$
b=b(n, i)= \begin{cases}0 & \text { if } n \geqslant i, \\ 1 & \text { if } n<i\end{cases}
$$

Then

$$
\begin{aligned}
A_{n} & =\sum_{\substack{t=1 \\
t \equiv n(\bmod p)}}^{f} \chi(t)=\sum_{a=0}^{g-1+b} \chi(p a+n) \\
& =\chi(p) \sum_{a=0}^{g-1+b} \chi\left(a+p^{-1} n\right)=\chi(p) \sum_{t=p^{-1} n}^{p^{-1} n+g-1+b} \chi(t),
\end{aligned}
$$

where $p^{-1}$ denotes $p^{-1}(\bmod f)$. Thus

$$
A_{0}=\chi(p) \sum_{t=0}^{g} \chi(t) \text {. }
$$

Since $i \equiv-p g(\bmod f)$,

$$
p^{-1}(p-i) \equiv p^{-1}(p+p g) \equiv 1+g(\bmod f),
$$

so it follows that

$$
A_{p-i}=\chi(p) \sum_{t=g+1}^{2 g+j} \chi(t) .
$$

If $2 i<p$, then $1 \leqslant p-2 i<p$ and

$$
p^{-1}(p-2 i) \equiv p^{-1}(p+2 p g) \equiv 2 g+1 \quad(\bmod f) \text {. }
$$

Thus

$$
A_{p-2 i}=\chi(p) \sum_{t=2 g+1}^{3 g+t} \chi(t) .
$$

If $2 i>p$, then $1<2(p-i)<p$ and

$$
2(p-i) p^{-1} \equiv 2(p+p g) p^{-1} \equiv 2 g+2 \quad(\bmod f) .
$$

Thus

$$
A_{2(p-i)}=\chi(p) \sum_{t=2 g+2}^{3 g+s+1} \chi(t) .
$$

THEOREM 3. Explicit conditions for each of the primes $p=3,5$ and 7 to be $m$-regular for $(m, p)=1$ are:

(a) $p=3$ if and only if $A_{0} \not \equiv 0(\bmod 3)$.

(b) $p=5$ and $m<0$ if and only if

$$
A_{4 f}\left(A_{0}+3 A_{4 f}\right) \neq \equiv 0 \quad(\bmod 5) \text {. }
$$

(c) $p=5$ and $m>0$ if and only if

$$
\left(A_{0}-A_{4 f}\right)\left(A_{0}+2 A_{4 f}\right) \not \equiv 0 \quad(\bmod 5) \text {. }
$$

(d) $p=7$ and $m<0$ if and only if

$$
\left(A_{5 f}+3 A_{6 f}\right)\left(A_{5 f}-2 A_{6 f}\right)\left(A_{0}-2 A_{5 f}+3 A_{6 f}\right) \not \equiv 0 \quad(\bmod 7) .
$$


(e) $p=7$ and $m>0$ if and only if

$$
\left(A_{0}-3 A_{5 f}+2 A_{6 f}\right)\left(A_{0}-A_{5 f}\right)\left(A_{0}-3 A_{5 f}+3 A_{6 f}\right) \not \equiv 0 \quad(\bmod 7) .
$$

Proof. Since the proof of each of the five parts is similar, we shall prove only part (e). Lemma 3 shows that $A_{0}=A_{f}, A_{2 f}=A_{6 f}$ and $A_{3 f}=A_{5 f}$, and since

$$
\sum_{j=0}^{6} A_{j f}=\sum_{t=1}^{f} \chi(t)=0
$$

then

$$
A_{4 f}=-2\left(A_{0}+A_{5 f}+A_{6 f}\right) \text {. }
$$

Applying Theorem 2 with $n=1,3$ and 5 shows that 7 is $m$-regular if and only if

$$
\begin{array}{ll}
A_{2 f}+3 A_{3 f}+6 A_{4 f}+3 A_{5 f}+A_{6 f} \not \equiv 0 & (\bmod 7), \\
A_{2 f}+2 A_{3 f}+A_{4 f}+2 A_{5 f}+A_{6 f} \not \equiv 0 & (\bmod 7), \\
A_{2 f}+5 A_{3 f}+3 A_{4 f}+5 A_{5 f}+A_{6 f} \not \equiv 0 & (\bmod 7) .
\end{array}
$$

Using the relations on the $A$ 's given above, the last three congruences reduce to the condition stated in (e).

Remarks. (1) The $A$ 's in Theorem 3 can easily be computed using Lemma 4.

(2) When $p=3, K$ is a bicyclic, biquadratic field, and the Dirichlet class number relation shows that 3 is $m$-regular if and only if each of the quadratic subfields has class number not divisible by 3. A result of Scholz [8] shows that the latter condition is satisfied if and only if the imaginary quadratic subfield $Q(\sqrt{m})$ or $Q(\sqrt{-3 m})$ of $K$ has class number relatively prime to 3. C. Queen [7] has shown the latter condition is equivalent to part (a) of Theorem 3 under the hypothesis that $m \equiv 1(\bmod 4)$ and $m$ is a positive prime.

For any real number $x$, let $[x]$ and $\{x\}$ denote the greatest integer less than or equal to $x$ and the least integer greater than or equal to $x$, respectively. Define

$$
S_{n}(x)=S_{n}(\{x\})=\sum_{u=0}^{\{x\}-1} u^{n}
$$

THEOREM 4. Let $p$ be an odd regular prime and $\chi$ the nontrivial character of the field $k=Q(\sqrt{m})$ with conductor $f$ where $(m, p)=1$. If $p>f$, then $p$ is m-regular if and only if

$$
\sum_{j=1}^{[(f-1) / 2]}\left(S_{n}\left(\frac{j p}{f}\right)-E\right) \chi(j) \not \equiv 0 \quad(\bmod p)
$$

for all $n$ with $1 \leqslant n \leqslant p-1$ and $n \equiv 1+\delta_{\chi}(\bmod 2)$. Here $E=(p-1) / 2$ or 0 according as $n=p-1$ or not.

Proof. According to Theorem 2, $p$ is $m$-regular if and only if

$$
\sum_{j=1}^{p} S_{n}(j) A_{j f} \neq 0 \quad(\bmod p)
$$


for all $n$ with $1 \leqslant n \leqslant p-1$ and $n \equiv 1+\delta_{\chi}(\bmod 2)$. If $j f \equiv f_{j}(\bmod p)$ with $0 \leqslant f_{j}<p$, then since $f<p$,

$$
A_{j f}=\sum_{\substack{t=1 \\ t=j f(\bmod p)}}^{f} \chi(t)= \begin{cases}\chi\left(f_{j}\right) & \text { if } f_{j}<f, \\ 0 & \text { if } f_{j} \geqslant f .\end{cases}
$$

If $f_{j}=k$, then $j \equiv f^{-1} k \equiv k_{f}(\bmod p)$, where $0 \leqslant k_{f}<p$. Thus

$$
\sum_{j=1}^{p} S_{n}(j) A_{j f}=\sum_{k=1}^{f-1} S_{n}\left(k_{f}\right) \chi(k) \text {. }
$$

Now if $j_{k} \equiv-p^{-1} k(\bmod f)$ with $0<j_{k}<f$, then $\left(j_{k} p+k\right) / f$ is an integer less than $p$ and $\left(j_{k} p+k\right) / f \equiv f^{-1} k(\bmod p)$. Thus $k_{f}=\left(j_{k} p+k\right) / f$ and so

$$
\begin{aligned}
\sum_{j=1}^{p} S_{n}(j) A_{j f} & =\sum_{k=1}^{f-1} S_{n}\left(\frac{j_{k} p+k}{f}\right) \chi(k)=\sum_{k=1}^{f-1} S_{n}\left(\frac{j_{k} p}{f}\right) \chi(k) \\
& =\sum_{j=1}^{f-1} S_{n}\left(\frac{j p}{f}\right) \chi(-p j)=\chi(-p) \sum_{j=1}^{f-1} S_{n}\left(\frac{j p}{f}\right) \chi(j) \\
& =\chi(-p) \sum_{j=1}^{[(f-1) / 2]}\left[S_{n}\left(\frac{j p}{f}\right)+\chi(-1) S_{n}\left(\frac{(f-j) p}{f}\right)\right] \chi(j) .
\end{aligned}
$$

Since $\sum_{u=1}^{p-1} u^{n} \equiv C(\bmod p)$, where $C=-1$ or 0 according as $n=p-1$ or not,

$$
\begin{aligned}
\chi(-1) S_{n}\left(\frac{(f-j) p}{f}\right) & \equiv-\chi(-1)\left(-C+\sum_{u=\{(f-j) p / f\}}^{p-1} u^{n}\right) \\
& \equiv-\chi(-1)\left(-C+\sum_{u=1}^{[j p / f]}(-u)^{n}\right) \equiv-C+\sum_{u=1}^{[j p / f]} u^{n} \\
& \equiv-C+S_{n}\left(\frac{j p}{f}\right) \quad(\bmod p),
\end{aligned}
$$

because $\chi$ and $n$ have opposite parity. Hence

$$
\sum_{j=1}^{p} S_{n}(j) A_{j f} \equiv \chi(-p) \sum_{j=1}^{[(f-1) / 2]}\left(2 S_{n}\left(\frac{j p}{f}\right)-C\right) \chi(j) \quad(\bmod p),
$$

and the desired result follows immediately from Theorem 2.

COROLlary. Explicit conditions for a regular prime $p>7$ to be m-regular for $m=-1, \pm 2,-3,5$ and -7 are:

(a) $m=-1$ :

$$
\sum_{0<u<p / 4} u^{n} \not \equiv 0 \quad(\bmod p)
$$

(b) $m=2$ :

$$
\sum_{p / 8<u<3 p / 8} u^{n} \equiv 0 \quad(\bmod p),
$$

(c) $m=-2$ :

$$
\sum_{0<u<p / 8} u^{n}-\sum_{3 p / 8<u<p / 2} u^{n} \not \equiv 0 \quad(\bmod p)
$$


(d) $m=-3$ :

$$
\sum_{0<u<p / 3} u^{n} \neq \equiv 0 \quad(\bmod p)
$$

(e) $m=5$ :

$$
\sum_{p / 5<u<2 p / 5} u^{n} \not \equiv 0 \quad(\bmod p)
$$

(f) $m=-7$ :

$$
\sum_{0<u<p / 7} u^{n}-\sum_{2 p / 7<u<3 p / 7} u^{n} \neq 0 \quad(\bmod p),
$$

where $1 \leqslant n \leqslant p-2$ and $n$ is even or odd according as $m$ is negative or positive.

Proof. As the proof of each part is similar, we shall prove only part (f). From Theorem $4, p$ is -7 -regular if and only if

$$
\sum_{j=1}^{3}\left(S_{n}\left(\frac{j p}{7}\right)-E\right) \chi(j) \not \equiv 0 \quad(\bmod p)
$$

for all even $n$ with $1 \leqslant n \leqslant p-1$. As seen in the proof of Theorem 2 , the condition for $n=p-1$ is equivalent to the fact that $h(Q(\sqrt{-7}))=1$ is not divisible by $p$. Thus we may assume $n<p-1$ and so $E=0$. The above condition becomes

$$
S_{n}\left(\frac{p}{7}\right)+S_{n}\left(\frac{2 p}{7}\right)-S_{n}\left(\frac{3 p}{7}\right) \not \equiv 0 \quad(\bmod p)
$$

for all even $n$ with $1<n<p-2$. Since

$$
S_{n}\left(\frac{2 p}{7}\right)-S_{n}\left(\frac{3 p}{7}\right)=-\sum_{2 p / 7<u<3 p / 7} u^{n},
$$

the proof of part (f) is complete.

Using Fortran programs, we did two different types of calculations. First, using the results of Theorem 3, we computed all values of $m$ with $m=n$ or $2 n$ and $|n|<10,000$ for which the primes $p=3,5$ or 7 are $m$-irregular. A summary of these results appears in Table 1 . Next we determine which primes $p<5025$ satisfy the hypothesis of the corollary to Theorem 4 for each $m=-3,-1,-7,-2,5$ and 2 . This computation gives exactly those primes $p$ for which $h^{*}=h^{*}(p, m)$ is divisible by $p$. Since any irregular prime is, by our definition, always $m$-irregular, a complete list of $m$-irregular primes can be obtained by including those irregular primes $p<5025$ which do not appear in Table 2. In [2], Ernvall and Metsänkylä have studied $m$-regular primes with $m=-1$. It is reassuring that there are no discrepancies between their table and ours.

TABLE 1: $m=n$ or $2 n$ with $|n|<10,000$

\begin{tabular}{|l|r|r|r|}
\hline \multicolumn{1}{|c|}{$p$} & 3 & 5 & 7 \\
\hline Number values $m$ & 12169 & 13515 & 14193 \\
\hline Number $m$-irregular & 4474 & 5202 & 5548 \\
\hline Percent $m$-irregular & 36.8 & 38.5 & 39.1 \\
\hline
\end{tabular}


TABLE 2: $m$-irregular primes $p \leqslant 5025$

\begin{tabular}{|c|c|c|c|c|c|}
\hline$p \backslash m \quad-3$ & -1 & -7 & -2 & 5 & 2 \\
\hline 23 & 19 & 29 & 19 & 17 & 11 \\
\hline 47 & 31 & 47 & 23 & 19 & 13 \\
\hline 53 & 43 & 53 & 59 & 41 & 19 \\
\hline 67 & 47 & 59 & 73 & 61 & 31 \\
\hline 103 & 61 & 73 & 83 & 67 & 37 \\
\hline 113 & 67 & 83 & 89 & 73 & 59 \\
\hline 139 & 71 & 109 & 113 & 107 & 71 \\
\hline 197 & 79 & 113 & 131 & 127 & 79 \\
\hline 199 & 101 & 137 & 137 & 131 & 89 \\
\hline 241 & 13.7 & 139 & 139 & 137 & 107 \\
\hline 257 & 139 & 149 & 149 & 139 & 127 \\
\hline 263 & 149 & 157 & 173 & 149 & 149 \\
\hline 271 & 193 & 173 & 179 & 151 & 151 \\
\hline 281 & 223 & 181 & 197 & 163 & 173 \\
\hline 317 & 241 & 191 & 223 & 167 & 179 \\
\hline 331 & 251 & 193 & 227 & 191 & 199 \\
\hline 337 & 263 & 223 & 229 & 239 & 229 \\
\hline 347 & 277 & 233 & 233 & 251 & 293 \\
\hline 353 & 307 & 263 & 241 & 281 & 307 \\
\hline 401 & 311 & 281 & 263 & 293 & 347 \\
\hline 409 & 349 & 307 & 269 & 313 & 359 \\
\hline 419 & 353. & 313 & 307 & 331 & 367 \\
\hline 421 & 359 & 317 & 317 & 347 & 379 \\
\hline 457 & 373 & 349 & 347 & 349 & 383 \\
\hline 467 & 379 & 353 & 353 & 383 & 397 \\
\hline 491 & 419 & 359 & 379 & 389 & 409 \\
\hline 521 & 433 & 373 & 397 & 401 & 439 \\
\hline 547 & 461 & 379 & 449 & 409 & 443 \\
\hline 577 & 463 & 383 & 457 & 439 & 479 \\
\hline 601 & 491 & 401 & 461 & 443 & 491 \\
\hline 617 & 509 & 421 & 463 & 449 & 499 \\
\hline 631 & 541 & 463 & 487 & 457 & 521 \\
\hline 643 & 563 & 467 & 523 & 499 & 541 \\
\hline 661 & 571 & 479 & 547 & 503 & 569 \\
\hline 673 & 577 & 499 & 601 & 547 & 571 \\
\hline 677 & 587 & 503 & 607 & 357 & 587 \\
\hline 683 & 619 & 521 & 613 & 569 & 593 \\
\hline 691 & 677 & 541 & 641 & 601 & 607 \\
\hline 809 & 691 & 547 & 643 & 617 & 617 \\
\hline 811 & 709 & 563 & 647 & 641 & 659 \\
\hline
\end{tabular}


TABLE 2 (continued)

\begin{tabular}{|c|c|c|c|c|c|}
\hline$p \backslash^{m}$ & -1 & -7 & -2 & 5 & 2 \\
\hline 821 & 739 & 587 & 653 & 653 & 691 \\
\hline 859 & 751 & 593 & 661 & 659 & 709 \\
\hline 863 & 761 & 607 & 673 & 661 & 739 \\
\hline 877 & 769 & 653 & 677 & 673 & 761 \\
\hline 887 & 773 & 659 & 683 & 677 & 787 \\
\hline 919 & 811 & 661 & 691 & 683 & 797 \\
\hline 997 & 821 & 691 & 739 & 701 & 809 \\
\hline 1009 & 877 & 701 & 757 & 769 & 821 \\
\hline 1013 & 887 & 709 & 811 & 787 & 823 \\
\hline 1039 & 907 & 751 & 823 & 821 & 853 \\
\hline 1049 & 929 & 787 & 853 & 839 & 859 \\
\hline 1061 & 941 & 797 & 857 & 863 & 911 \\
\hline 1091 & 967 & 823 & 883 & 941 & 919 \\
\hline 1093 & 971 & 827 & 953 & 953 & 929 \\
\hline 1097 & 983 & 863 & 977 & 977 & 937 \\
\hline 1151 & 1013 & 877 & 983 & 1009 & 953 \\
\hline 1153 & 1019 & 911 & 997 & 1019 & 977 \\
\hline 1171 & 1031 & 919 & 1019 & 1039 & 983 \\
\hline 1187 & 1039 & 947 & 1031 & 1051 & 991 \\
\hline 1193 & 1049 & 967 & 1033 & 1063 & 1013 \\
\hline 1201 & 1051 & 977 & 1063 & 1087 & 1019 \\
\hline 1213 & 1069 & 997 & 1069 & 1093 & 1087 \\
\hline 1217 & 1151 & 1009 & 1087 & 1109 & 1091 \\
\hline 1237 & 1163 & 1031 & 1093 & 1129 & 1093 \\
\hline 1249 & 1187 & 1051 & 1097 & 1151 & 1097 \\
\hline 1283 & 1223 & 1061 & 1129 & 1181 & 1103 \\
\hline 1297 & 1229 & 1087 & 1151 & 1201 & 1129 \\
\hline 1303 & 1231 & 1097 & 1163 & 1213 & 1151 \\
\hline 1319 & 1277 & 1117 & 1181 & 1237 & 1187 \\
\hline 1409 & 1279 & 1129 & 1187 & 1249 & 1193 \\
\hline 1453 & 1283 & 1153 & 1213 & 1277 & 1217 \\
\hline 1523 & 1291 & 1171 & 1291 & 1283 & 1223 \\
\hline 1567 & 1307 & 1201 & 1303 & 1289 & 1229 \\
\hline 1579 & 1319 & 1231 & 1319 & 1291 & 1289 \\
\hline 1583 & 1361 & 1283 & 1327 & 1303 & 1291 \\
\hline 1607 & 1381 & 1409 & 1361 & 1327 & 1307 \\
\hline 1013 & 1399 & 1439 & 1381 & 1433 & 1321 \\
\hline 1621 & 1409 & 1451 & 1427 & 1447 & 1381 \\
\hline 1663 & 1423 & 1453 & 1429 & 1511 & 1423 \\
\hline 1667 & 1427 & 1459 & 1439 & 1523 & 1427 \\
\hline
\end{tabular}


TABLE 2 (continued)

\begin{tabular}{|c|c|c|c|c|c|}
\hline$p \backslash^{m}$ & -1 & -7 & -2 & 5 & 2 \\
\hline 1669 & 1429 & 1471 & 1447 & 1543 & 1433 \\
\hline 1697 & 1439 & 1481 & 1451 & 1601 & 1439 \\
\hline 1699 & 1447 & 1483 & 1453 & 1607 & 1453 \\
\hline 1709 & 1453 & 1523 & 1489 & 1619 & 1481 \\
\hline 1733 & 1523 & 1531 & 1499 & 1627 & 1483 \\
\hline 1759 & 1531 & 1549 & 1511 & 1637 & 1489 \\
\hline 1777 & 1559 & 1553 & 1549 & 1693 & 1493 \\
\hline 1823 & 1583 & 1559 & 1567 & 1733 & 1499 \\
\hline 1847 & 1601 & 1597 & 1571 & 1741 & 1559 \\
\hline 1867 & 1621 & 1607 & 1579 & 1747 & 1567 \\
\hline 1871 & 1637 & 1627 & 1583 & 1783 & 1571 \\
\hline 1901 & 1663 & 1657 & 1601 & 1823 & 1579 \\
\hline 1913 & 1693 & 1667 & 1607 & 1867 & 1607 \\
\hline 1951 & 1697 & 1669 & 1609 & 1873 & 1609 \\
\hline 1973 & 1723 & 1699 & 1613 & 1879 & 1637 \\
\hline 1979 & 1733 & 1723 & 1619 & 1931 & 1667 \\
\hline 1993 & 1759 & 1783 & 1637 & 1933 & 1669 \\
\hline וו1 & 1787 & 1787 & 1657 & 1973 & 1693 \\
\hline 2063 & 1801 & 1801 & 1667 & 1987 & 1697 \\
\hline 2069 & 1831 & 1811 & 1669 & 1999 & 1721 \\
\hline 2083 & 1867 & 1823 & 1697 & 2003 & 1759 \\
\hline 2089 & 1873 & 1831 & 1699 & 2017 & 1777 \\
\hline 2099 & 1877 & 1871 & 1721 & 2029 & 1783 \\
\hline 2131 & 1879 & 1877 & 1753 & 2053 & 1787 \\
\hline 2161 & 1889 & 1879 & 1823 & 2063 & 1823 \\
\hline 2203 & 1901 & 1907 & 1861 & 2081 & 1867 \\
\hline 2207 & 1907 & 1913 & 1873 & 2087 & 1873 \\
\hline 2221 & 1931 & 1931 & 1907 & 2111 & 1889 \\
\hline 2239 & 1933 & 1933 & 1949 & 2137 & 1931 \\
\hline 2269 & 1951 & 1993 & 1979 & 2141 & 1993 \\
\hline 2281 & 1987 & 1999 & 1987 & 2143 & 2003 \\
\hline 2293 & 1993 & 2003 & 1997 & 2161 & 2011 \\
\hline 2309 & 1997 & 2017 & 2003 & 2179 & 2053 \\
\hline 2347 & 2011 & 2027 & 2011 & 2207 & 2063 \\
\hline 2351 & 2039 & 2029 & 2017 & 2239 & 2087 \\
\hline 2441 & 2063 & 2053 & 2039 & 2243 & 2741 \\
\hline 2473 & 2069 & 2063 & 2069 & 2267 & 2143 \\
\hline 2477 & 2081 & 2069 & 2081 & 2287 & 2221 \\
\hline 2531 & 2083 & 2081 & 2089 & 2297 & 2239 \\
\hline 2539 & 2099 & 2111 & 211 & 2339 & 2243 \\
\hline
\end{tabular}


TABLE 2 (continued)

\begin{tabular}{|c|c|c|c|c|c|}
\hline$p\rangle^{m}$ & -1 & -7 & -2 & 5 & 2 \\
\hline 2549 & 2129 & 2203 & 2129 & 2341 & 2251 \\
\hline 2557 & 2131 & 2221 & 2137 & 2351 & 2267 \\
\hline 2609 & 2137 & 2237 & 2153 & 2371 & 2273 \\
\hline 2621 & 2141 & 2269 & 2203 & 2381 & 2287 \\
\hline 2647 & 2143 & 2281 & 2243 & 2383 & 2339 \\
\hline 2663 & 2161 & 2309 & 2251 & 2423 & 2347 \\
\hline I267 & 2179 & 2377 & 2281 & 2441 & 2371 \\
\hline 2693 & 2203 & 2381 & 2297 & 2467 & 2399 \\
\hline 2699 & 2213 & 2383 & 2333 & 2477 & 2411 \\
\hline 2707 & 2221 & 2389 & 2339 & 2503 & 2417 \\
\hline 2749 & 2239 & 2393 & 2341 & 2521 & 2447 \\
\hline 2767 & 2293 & 2399 & 2371 & 2531 & 2459 \\
\hline 2797 & 2341 & 2423 & 2393 & 2543 & 2473 \\
\hline 2803 & 2377 & 2441 & 2399 & 2551 & 2521 \\
\hline 2819 & 2411 & 2473 & 2411 & 2609 & 2549 \\
\hline 2833 & 2417 & 2521 & 2441 & 2617 & 2579 \\
\hline 2843 & 2459 & 2531 & 2467 & 2621 & 2591 \\
\hline 2851 & 2473 & 2549 & 2477 & 2663 & 2617 \\
\hline 2879 & 2477 & 2579 & 2503 & ווו & 2621 \\
\hline 2903 & 2531 & 2591 & 2521 & 2713 & 2633 \\
\hline 2939 & 2543 & 2609 & 2539 & 2749 & 2663 \\
\hline 2963 & 2579 & 2633 & 2579 & 2753 & 2671 \\
\hline 2969 & 2591 & 2647 & 2647 & 2791 & 2677 \\
\hline 2971 & 2609 & 2677 & 2659 & 2819 & 2689 \\
\hline 2999 & 2617 & 2687 & 2671 & 2833 & 2699 \\
\hline 3011 & 2633 & 271 & 2741 & 2837 & 2741 \\
\hline 3023 & 2659 & 2731 & 2753 & 2843 & 2753 \\
\hline 3041 & 2671 & 2767 & 2767 & 2851 & 2801 \\
\hline 3049 & 2677 & 2777 & 2789 & 2879 & 2803 \\
\hline 3061 & 2687 & 2801 & 2791 & 2897 & 28443 \\
\hline 3067 & 2699 & 2861 & 2833 & 2903 & 2879 \\
\hline 3083 & וו1 & 2897 & 2857 & 2927 & 2807 \\
\hline 3089 & 2729 & 2909 & 2861 & 2957 & 2903 \\
\hline 3121 & 2731 & 2917 & 2887 & 2969 & $2 \subseteq 09$ \\
\hline 3181 & 2749 & 2927 & 2917 & 2999 & 2917 \\
\hline 3217 & 2797 & 2971 & 2927 & 3011 & 2957 \\
\hline 3229 & 2803 & 3001 & 2939 & 3049 & 2963 \\
\hline 3251 & 2819 & 3011 & 2953 & 3061 & 2971 \\
\hline 3259 & 2843 & 3019 & 2999 & 3067 & 3001 \\
\hline 3301 & 2879 & 3023 & 3019 & 3083 & 3041 \\
\hline
\end{tabular}


TABLE 2 (continued)

\begin{tabular}{|c|c|c|c|c|c|}
\hline$p\rangle^{m}$ & -1 & -7 & -2 & 5 & 2 \\
\hline 3319 & 2897 & 3037 & 3037 & 3109 & 3049 \\
\hline 3329 & 2917 & 3083 & 3041 & 3181 & 3061 \\
\hline 3343 & 2957 & 3121 & 3119 & 3209 & 3067 \\
\hline 3361 & 2963 & 3137 & 3121 & 3251 & 3167 \\
\hline 3373 & 2971 & 3203 & 3191 & 3253 & 3209 \\
\hline 3407 & 2999 & 3271 & 3217 & 3257 & 3217 \\
\hline 3413 & 3001 & 3299 & 3221 & 3271 & 3229 \\
\hline 3461 & 3061 & 3301 & 3251 & 3307 & 3253 \\
\hline 3463 & 3067 & 3331 & 3253 & 3313 & 3271 \\
\hline 3499 & 3079 & 3347 & 3259 & 3319 & 3301 \\
\hline 3533 & 3089 & 3359 & 3271 & 3329 & 3313 \\
\hline 3539 & 3119 & 3361 & 3313 & 3347 & 3343 \\
\hline 3541 & 3121 & 3391 & 3323 & 3359 & 3347 \\
\hline 3581 & 3137 & 3407 & 3331 & 3371 & 3361 \\
\hline 3583 & 3163 & 3449 & 3343 & 3407 & 3389 \\
\hline 3631 & 3167 & 3469 & 3361 & 3461 & 3391 \\
\hline 3637 & 3169 & 3499 & 3391 & 3467 & 3457 \\
\hline 3643 & 3187 & 3529 & 3407 & 3469 & 3469 \\
\hline 3659 & 3217 & 3533 & 3433 & 3511 & 3499 \\
\hline 3673 & 3257 & 3547 & 3449 & 3517 & 3511 \\
\hline 3677 & 3301 & 3559 & 3511 & 3529 & 3529 \\
\hline 3691 & 3313 & 3571 & 3557 & 3559 & 3533 \\
\hline 3727 & 3331 & 3583 & 3559 & 3607 & 3559 \\
\hline 3733 & 3343 & 3593 & 3571 & 3613 & 3581 \\
\hline 3761 & 3449 & 3613 & 3581 & 3617 & 3613 \\
\hline 3797 & 3467 & 3631 & 3607 & 3643 & 3623 \\
\hline 3821 & 3491 & 3637 & 3623 & 3673 & 3659 \\
\hline 3823 & 3517 & 3709 & 3643 & 3677 & 3671 \\
\hline 3833 & 3539 & 3727 & 3659 & 3691 & 3673 \\
\hline 3853 & 3541 & 3779 & 3673 & 3697 & 3677 \\
\hline 3907 & 3547 & 3821 & 3719 & 3701 & 3691 \\
\hline 3931 & 3571 & 3833 & 3761 & 3719 & 3697 \\
\hline 3947 & 3581 & 3851 & 3767 & 3727 & 3709 \\
\hline 3967 & 3623 & 3853 & 3769 & 3733 & 3727 \\
\hline 4007 & 3631 & 3863 & 3779 & 3761 & 3739 \\
\hline 4013 & 3671 & 3911 & 3821 & 3779 & 3767 \\
\hline 4021 & 3673 & 3929 & 3851 & 3803 & 3779 \\
\hline 4027 & 3677 & 3943 & 3863 & 3821 & 3793 \\
\hline 4093 & 3701 & 3967 & 3881 & 3853 & 3797 \\
\hline 4127 & 3727 & 4007 & 3917 & 3881 & 3821 \\
\hline
\end{tabular}


TABLE 2 (continued)

\begin{tabular}{|c|c|c|c|c|c|}
\hline$p \backslash^{m}$ & -1 & -7 & -2 & 5 & 2 \\
\hline 4153 & 3733 & 4019 & 3919 & 3889 & 3863 \\
\hline 4157 & 3761 & 4021 & 3931 & 3911 & 3889 \\
\hline 4219 & 3793 & 4027 & 3943 & 3917 & 3907 \\
\hline 4231 & 3797 & 4057 & 4003 & 3919 & 3919 \\
\hline 4259 & 3821 & 4073 & 4021 & 3943 & 3947 \\
\hline 4261 & 3833 & 4091 & 4051 & 3947 & 3967 \\
\hline 4297 & 3847 & 411 & 4079 & 3967 & 4007 \\
\hline 4337 & 3851 & 4129 & 4099 & 3989 & 4019 \\
\hline 4357 & 3853 & 4133 & 4111 & 4013 & 4073 \\
\hline 4441 & 3911 & 4159 & 4133 & 4019 & 4093 \\
\hline 4447 & 3917 & 4231 & 4219 & 4027 & 4099 \\
\hline 4451 & 3923 & 4241 & 4231 & 4051 & ווו14 \\
\hline 4481 & 3989 & 4259 & 4241 & 4093 & 4129 \\
\hline 4483 & 4003 & 4261 & 4243 & 4099 & 4153 \\
\hline 4493 & 4007 & 4339 & 4253 & 4153 & 4159 \\
\hline 4517 & 4021 & 4349 & 4261 & 4211 & 4177 \\
\hline 4519 & 4051 & 4363 & 4273 & 4241 & 4201 \\
\hline 4523 & 4057 & 4373 & 4289 & 4253 & 4217 \\
\hline 4547 & 4093 & 4441 & 4357 & 4283 & 4241 \\
\hline 4567 & 4099 & 4447 & 4423 & 4327 & 4271 \\
\hline 4583 & 4129 & 4451 & 4447 & 4337 & 4297 \\
\hline 4597 & 4133 & 4463 & 4481 & 4357 & 4339 \\
\hline 4643 & 1153 & 4481 & 4483 & 4373 & 4397 \\
\hline 4649 & 4241 & 4493 & 4493 & 4451 & 4421 \\
\hline 4651 & 4259 & 4513 & 4507 & 4457 & 4423 \\
\hline 4657 & 4271 & 4517 & 4519 & 4463 & 4447 \\
\hline 4813 & 4283 & 4547 & 4549 & 4481 & 4493 \\
\hline 4831 & 4289 & 4583 & 4561 & 4519 & 4507 \\
\hline 4903 & 4337 & 4597 & 4567 & 4523 & 4523 \\
\hline 4919 & 4339 & 4621 & 4583 & 4549 & 4561 \\
\hline 4969 & 4349 & 4639 & 4597 & 4643 & 4591 \\
\hline 4993 & 4357 & 4663 & 4621 & 4649 & 4597 \\
\hline 5003 & 4373 & 4679 & 4637 & 4651 & 4603 \\
\hline 5009 & 4391 & 4783 & 4639 & 4703 & 4639 \\
\hline \multirow[t]{6}{*}{5021} & 4397 & 4787 & 4643 & 4723 & 4657 \\
\hline & 4421 & 4831 & 4663 & 4789 & 4729 \\
\hline & 4463 & 4861 & 4679 & 4793 & 4787 \\
\hline & 4481 & 4903 & 4691 & 4861 & 4789 \\
\hline & 4493 & 4931 & 4721 & 4871 & 4793 \\
\hline & 4523 & 4933 & 4733 & 4903 & 4801 \\
\hline
\end{tabular}


TABLE 2 (continued)

\begin{tabular}{|c|c|c|c|c|c|}
\hline$p\rangle^{m} \quad-s$ & -1 & -7 & -2 & 5 & 2 \\
\hline & 4549 & 4943 & 4751 & 4919 & 4817 \\
\hline & 4591 & 4993 & 4759 & 4931 & 4861 \\
\hline & 4603 & 5003 & 4789 & 4937 & 4871 \\
\hline & 4643 & 5011 & 4889 & 4943 & 4889 \\
\hline & 4657 & 5023 & 4931 & 4957 & 4919 \\
\hline & 4673 & & 4951 & 4967 & 4931 \\
\hline & 4679 & & 4969 & 5009 & 4937 \\
\hline & 4691 & & 4973 & 5011 & 4967 \\
\hline & 4703 & & 4993 & & 4993 \\
\hline & 4721 & & 5009 & & 5021 \\
\hline & 4729 & & 5011 & & \\
\hline & 4733 & & 5021 & & \\
\hline & 4789 & & 5023 & & \\
\hline & 4799 & & & & \\
\hline & 4813 & & & & \\
\hline & 4817 & & & & \\
\hline & 4861 & & & & \\
\hline & 4871 & & & & \\
\hline & 4933 & & & & \\
\hline & 4937 & & & & \\
\hline & 4943 & & & & \\
\hline & 5009 & & & & \\
\hline
\end{tabular}

Department of Mathematics

Valparaiso University

Valparaiso, Indiana 46383

Department of Mathematics

Virginia Polytechnic Institute and State University

Blacksburg, Virginia 24061

1. L. CaruITZ, "Arithmetic properties of generalized Bernoulli numbers," J. Reine Angew. Math., v. 202, 1959, pp. 174-182.

2. R. ERnVAll \& T. MetsänKYlä, "Cyclotomic invariants and $E$-irregular primes," Math. Comp., v. 32, 1978, pp. 617-629.

3. F. HAO \& C. PARRY, "The Fermat equation over quadratic fields," J. Number Theory. (To appear.)

4. H. HASSE, Über die Klassenzahl abelscher Zahlkörper, Akademie-Verlag, Berlin, 1952.

5. K. IwaSA Wa, Lectures on p-Adic L-Functions, Princeton Univ. Press, Princeton, N. J., 1972.

6. C. Parry, "On the class numbers of relative quadratic fields," Math. Comp., v. 32, 1978, pp. 1261-1270.

7. C. QUEEN, "A note on class numbers of imaginary quadratic number fields," Arch. Math. (Basel), v. 27, 1976, pp. 295-298.

8. A. Scholz, "Über die Beziehung der Klassenzahlen quadratischer Körper zueinander," J. Reine Angew: Math., v. 166, 1932, pp. 201-203.

9. L. Washington, Introduction to Cyclotomic Fields, Springer-Verlag, New York, Heidelberg, Berlin, 1982. 